\title{
Body Fat Distribution as a Risk Factor for Cerebrovascular Disease: An MRI-Based Body Fat Quantification Study
}

\author{
Hanna-Sofia Karcher Robert Holzwarth Hans-Peter Mueller Albert C. Ludolph \\ Roman Huber Jan Kassubek Elmar H. Pinkhardt \\ Department of Neurology, University of UIm, Ulm, Germany
}

\begin{abstract}
Key Words
Stroke - Obesity $\cdot$ Magnetic resonance imaging .

Cerebrovascular disease $\cdot$ Microangiopathy $\cdot$ Computed

tomography angiography $\cdot$ Risk factors for stroke
\end{abstract}

\begin{abstract}
Background: While adiposity is a well-established risk factor for cardiovascular disease, the association between adiposity and cerebrovascular disease is not entirely understood. For example, common methods to quantify body fat volume such as body mass index, waist circumference and waist-to-hip ratio are not suitable to identify the complex distribution patterns of body fat and its relation to cerebrovascular pathology. In view of a better understanding of the association between fat distribution and cerebrovascular disorders, the aim of the study was to perform measurements of body fat distribution patterns and body fat volumes in correlation to arteriosclerosis of the brain-feeding arteries and white matter lesion load (WMLL). Methods: In this study we performed a magnetic resonance imaging (MRI)-based volumetric differential analysis of subcutaneous and visceral body fat distribution in 25 patients with MRI-proven hyperacute ischemic stroke. For the measurement of adipose tissue volume and tissue distribution automatic labeling analysis software was
\end{abstract}

H.-S. Karcher and R. Holzwarth contributed equally to this work.

\section{KARGER}

(C) 2013 S. Karger AG, Basel

$1015-9770 / 13 / 0354-0341 \$ 38.00 / 0$

E-Mail karger@karger.com

www.karger.com/ced used. A correlation analysis of MRI volumetric measurements of subcutaneous and visceral body fat, atherosclerotic plaque load of the brain-feeding arteries measured by computed tomography angiography, and WMLL measured by MRI volumetry of the whole brain was performed. Results: The normalized total abdominal adipose tissue and the normalized subcutaneous abdominal adipose tissue showed no significant correlation with either WMLL or total plaque volume. In contrast, the normalized visceral adipose tissue showed a significant correlation with WMLL volume. Visceral adipose tissue as a percentage of total adipose tissue showed a significant correlation with WMLL. In particular, the percentage of visceral adipose tissue rather than total body fat volume strongly correlated with atherosclerosis and ischemic cerebral lesions. Furthermore, the volume of both soft and calcified plaques correlated significantly with WMLL. Conclusions: Our results contribute to existing studies about the association of different patterns of fat distribution with atherosclerosis of the brain-feeding arteries, in particular highlighting the importance of visceral adiposity as a risk factor for cerebrovascular disease. The percentage of visceral adipose tissue in total adipose tissue has the potential of a sensitive parameter and might become a relevant new epidemiological marker, showing highly significant correlations with well-established markers of cerebrovascular disease. In conclusion, the percentage of visceral adipose tissue by itself has to be regarded as a risk factor for both small vessel cerebrovascular disease and cerebral atherosclerosis of the large-to-medium-sized arteries.

Copyright $\odot 2013$ S. Karger AG, Basel

PD Dr. Elmar H. Pinkhardt, MD

Department of Neurology, University of Ulm

Oberer Eselsberg 45

DE-89081 Ulm (Germany)

E-Mail elmar.pinkhardt@uni-ulm.de 


\section{Introduction}

Cerebral stroke due to small vessel cerebrovascular disease and cerebral atherosclerosis of the large-to-mediumsized arteries are among the main reasons for severe neurological impairment in the elderly [1]. Nevertheless, risk factors for cerebrovascular disorders are not as well defined as in the case with cardiovascular disease (CVD) [2]. Obesity, as one of the main cardiovascular risk factors [3], has to be subdivided. Visceral obesity involves a high risk of CVD and is associated with an atherothrombotic, inflammatory profile of the metabolic syndrome [4]. It has been proposed that visceral fat may act like an 'endocrine gland'. The release of cytokines (adipokines) like TNFa facilitates the development of an insulin-resistant state, probably leading to higher CVD risk [5] and atherosclerosis [6]. Furthermore, free fatty acids, produced by visceral adipose tissue and released into the hepatic circulation, may influence lipoprotein metabolism and glucose homeostasis, thereby reducing insulin sensitivity [7]. On the other hand, peripheral subcutaneous fat, particularly located on the lower body, may be protective in respect to CVD [4].

In order to analyze fat distribution and its association with hypertension and stroke, the body mass index (BMI), measurements of waist circumference, waist-to-hip ratio and waist-to-height ratio have been performed. Apparently all these approaches to identify complex distribution patterns of body fat and their effects on cerebrovascular disease yield heterogeneous results. Only in some studies was BMI associated with a higher risk for stroke [8]; in other studies there was no significant association [9]. Winter et al. [10] found significant associations between the risk of stroke and waist circumference, waist-to-stature ratio and waist-to-hip ratio. Other studies showed that an increased waist-to-hip ratio was associated with greater risk of stroke and had a stronger association with stroke than BMI [11].

In regard to the differing risk profiles of fat tissues concerning CVD, a better understanding of the association between fat distribution and cerebrovascular disorders may help to reduce metabolic risk factor burden for cerebrovascular disease. Hence the aim of our study was (1) to perform measurements of body fat volumes and of distribution patterns of visceral and subcutaneous body fat in patients with cerebrovascular disease using a new magnetic resonance imaging (MRI)-based technique and (2) to analyze these results in relation to comorbidity with atherosclerotic changes of the brain-feeding arteries and white matter lesion load (WMLL).

\section{Materials and Methods}

\section{Study Population}

From October 2010 to January 2012, 25 patients with MRIproven hyperacute ischemic stroke ( 17 male, 8 female; average age, 65.7 years \pm 10.9 for men and 60.8 years \pm 13.3 for women) were enrolled from the stroke unit of the Department of Neurology, University of Ulm, Germany. All patients had undergone MRI of the brain and computed tomography angiography (CTA) of the brain-feeding arteries for clinical diagnostic purposes. In addition, a whole-body MRI scan for the evaluation of the quantity and distribution of adipose tissue was performed on all patients as part of the research protocol. The investigation was approved by the Ethics Committee of the University of Ulm, and all subjects gave their written informed consent before being enrolled.

\section{Data Recording and Data Processing}

CTA of the Supra-Aortic Vessels

CTA scanning of the ascending aorta, aortic arch and supraaortic vessels was performed using a 16-slice multidetector row CT system (Somatom Emotion; Siemens Healthcare, Erlangen, Germany). The following scanning protocol was used: spiral mode, $600 \mathrm{~ms}$ gantry rotation; collimation, $16 \times 0,6 \mathrm{~mm}$; pitch, 0.8 ; section thickness, $0,75 \mathrm{~mm}$; reconstruction interval, $0.75 \mathrm{~mm}$; acquisition parameters, $110 \mathrm{kVp} / 150 \mathrm{~mA}$. Patients received $120 \mathrm{ml}$ of a contrast agent (Imeron $400 \mathrm{MCT}$, Bracco Imaging $\mathrm{GmbH}$, Konstanz, Germany) using an injector with a flow rate of $3.5 \mathrm{ml} / \mathrm{s}$. Image reconstruction was carried out with a field of view of $187 \mathrm{~mm}$ and a slice thickness of $1 \mathrm{~mm}$.

Measurement of carotid artery wall thickness (CAWT) was performed as described previously by Saba et al. [12] by the use of the freely available DICOM software, OsiriX (OsiriX, version 3.7.1 for MacOS X). CAWT, defined as the distance between the leading edge of the opacified vessel lumen and the external visible limit of the artery wall [12] in axial CTA slices, was assessed for both common carotid arteries and was measured $1 \mathrm{~cm}$ proximal to the carotid bulb at areas where there was no evidence of a plaque.

The volume and distribution of atherosclerotic plaques was measured semiautomatically as a function of the threshold value of hyperdense areas with the in-house developed software, IBAS (Intensity-Based Analysis Software) [13]. Calcified plaques (fig. 1a) and fibrous and/or fatty plaques were delineated separately. The following vessels were analyzed: ascending aorta with aortic arch and the brachiocephalic artery; common carotid artery; extracranial internal carotid artery; intracranial internal carotid artery; anterior and medial cerebral artery; vertebral artery, and basilar and posterior cerebral artery.

\section{MRI Volumetric Measurement of WMLL}

Cranial MRI data were acquired on a 1.5-T scanner (Symphony; Siemens Healthcare) to quantify WMLL. Forty T2-weighted coronal slices (attenuated inversion recovery/fluid-attenuated inversion recovery, FLAIR, repetition time/echo time 6180/112 ms) of $3.0 \mathrm{~mm}$ thickness, $0,45 \times 0.45 \mathrm{~mm}^{2}$ in-plane resolution and $512 \times 448$ voxels matrix dimension were scanned. The $3 \mathrm{D}$ T2weighted images were analyzed by tensor-imaging and fiber-tracking software [14], based on a semiautomatic image intensity analysis. In order to identify lesion-related voxels (hyperintense in T2weighted scans), those voxels within an operator-defined intensity range were identified slicewise and were then color-coded depend- 

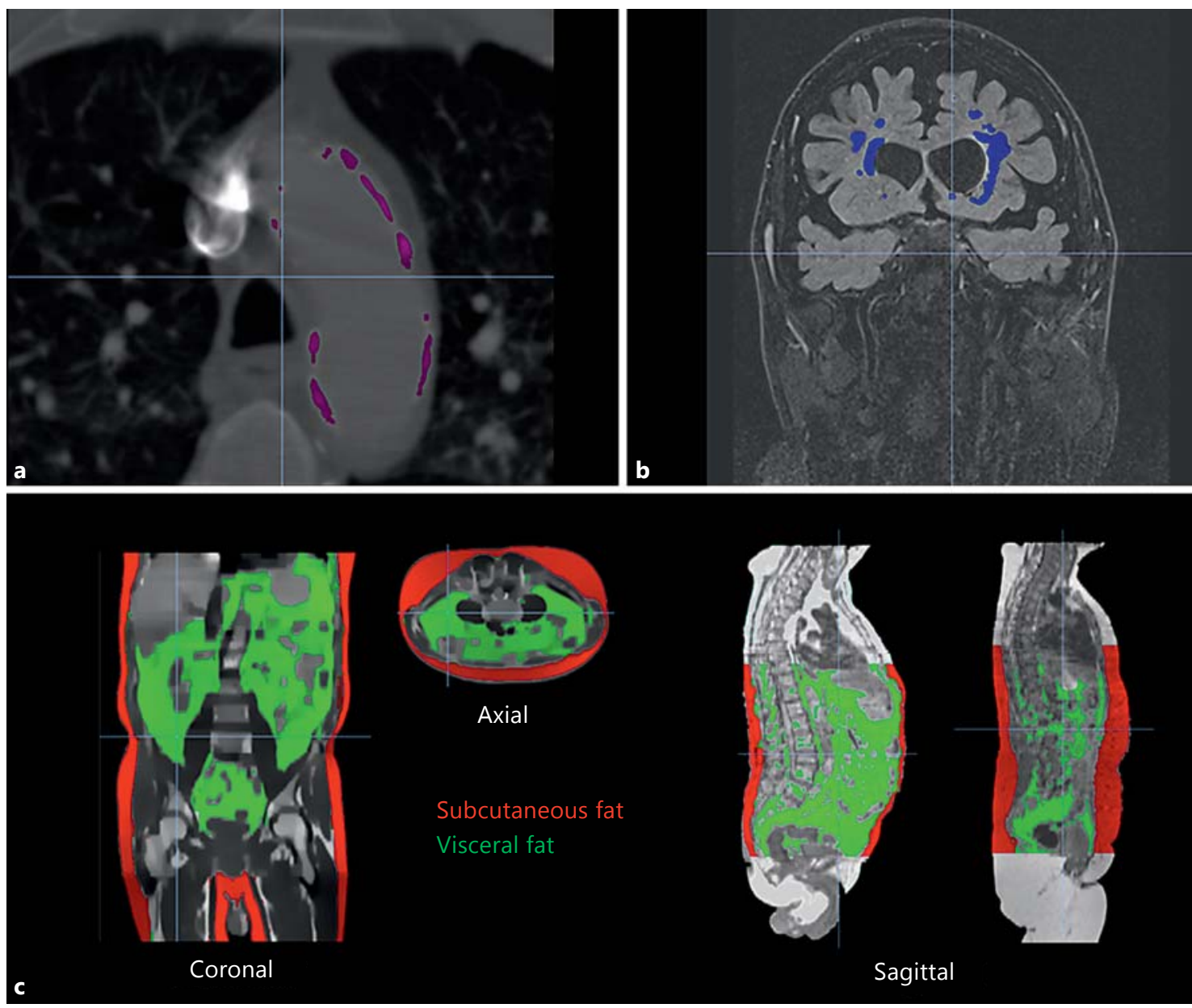

Fig. 1. a Quantification of atherosclerotic plaques in the aortic arch in CTA; hard (calcified) plaques are indicated in purple. b Determination of WMLL in a FLAIR MRI coronal slice; the measured white matter lesions are colored blue. c Measurement of body fat volumes; in representative slices (coronal, axial and sagittal view)
SAT is indicated in red and VAT in green. The sagittal view shows slices from 2 different subjects: the subject on the left has a high percentage of VAT and the subject on the right has a low percentage of VAT. ing on the anatomical localization (fig. 1b). A zooming feature using nearest neighbor interpolation improved the accuracy of this classification. The number of detected voxels, respectively the total volume of lesions, was identified as the WMLL.

\section{MRI Volumetric Analysis of Fat Volume and Distribution}

For body fat quantification, MRI data were acquired on the same 1.5-T scanner (Symphony; Siemens Healthcare). A quantum gradient system with gradient field strength up to $30 \mathrm{mT} / \mathrm{m}$ (52 $\mathrm{mT} / \mathrm{m}$ effective) was available with a slew rate of up to $125 \mathrm{~T} / \mathrm{m} / \mathrm{s}$ $(216 \mathrm{~T} / \mathrm{m} / \mathrm{s}$ effective). The whole-body MRI scan was recorded by the acquisition of six to eight T1-weighted volumes (standard T1weighted turbo spin echo sequence), each consisting of $362 \mathrm{D}$ slices (slice thickness, $5 \mathrm{~mm}$; $1 \mathrm{~mm}$ gap). The in-plane resolution was $1.8 \times 1.3 \mathrm{~mm}(206 \times 384$ voxels $) ; 206$ phase-encoding steps were used and the phase-encoding direction was anterior to posterior. The flip angle was $160^{\circ}$, the repetition time was $476 \mathrm{~ms}$ and the echo time was $12 \mathrm{~ms}$. The acquisition time for one volume was 4.5 min, resulting in a total acquisition time between 27 and $36 \mathrm{~min}$. To confirm that no gap was left between the consecutive volumes, an overlap of about 6-18 $\mathrm{mm}$ was chosen between the volumes, so that a total area of at least $120 \mathrm{~cm}$ was scanned.

For the measurement of adipose tissue volume and distribution, the in-house developed software package, ATLAS (Automatic Tissue Labeling Analysis Software) was used, as described previously [13]. An example of the measurement method is depicted in figure 1c. The region between the femoral head and the diaphragm was selected and defined as the abdominal region. Subcutaneous (SAT), visceral (intra-abdominal) adipose tissue (VAT) and total abdominal adipose tissue (TAT) was measured within these borders. In order to control for height differences, normalization of body fat volumes was performed by dividing each of the three volumes by the number of MRI slices used for the evaluation of body fat in each patient. 
Table 1. Volumetric analyses of white matter lesions, body fat and arterial wall thickness (mean $\pm \mathrm{SD}$ )

\begin{tabular}{lccc}
\hline & All $(\mathrm{n}=25)$ & Female $(\mathrm{n}=8)$ & Male $(\mathrm{n}=17)$ \\
\hline Age, years & $64.1 \pm 11.6$ & $60.8 \pm 13.3$ & $65.7 \pm 10.9$ \\
TPV, mm & $1,211 \pm 1,426$ & $677 \pm 937$ & $1,463 \pm 1,567$ \\
TAT, liters & $14.7 \pm 5.8$ & $14.9 \pm 8.6$ & $14.7 \pm 4.3$ \\
SAT, liters & $8.6 \pm 5.0$ & $11.0 \pm 7.4$ & $7.4 \pm 3.1$ \\
VAT, liters & $6.2 \pm 2.6$ & $4.0 \pm 1.7$ & $7.3 \pm 2.4$ \\
VAT/TAT & $0.435 \pm 0.135$ & $0.292 \pm 0.084$ & $0.502 \pm 0.097$ \\
nTAT, $1 / \mathrm{m}$ & $45.7 \pm 15.5$ & $46.3 \pm 23.1$ & $45.4 \pm 11.0$ \\
nSAT, $1 / \mathrm{m}$ & $26.3 \pm 13.6$ & $33.7 \pm 19.8$ & $22.7 \pm 7.9$ \\
nVAT, $1 / \mathrm{m}$ & $19.5 \pm 8.3$ & $12.6 \pm 5.3$ & $22.7 \pm 7.4$ \\
WMLL, mm & $6,379 \pm 7,037$ & $3,787 \pm 4,436$ & $7,599 \pm 7,793$ \\
CAWT, mm & $1.20 \pm 0.14$ & $1.10 \pm 0.12$ & $1.25 \pm 0.12$ \\
\hline
\end{tabular}

\section{Statistical Analysis}

Statistical analysis was done using IBM SPSS Statistics 20. Data are presented as mean \pm SD. Pearson's correlation coefficient was calculated to analyze the relation between the following variables: normalized total body fat (nTAT) with WMLL; nTAT with total plaque volume (TPV); normalized SAT (nSAT) with WMLL; nSAT with TPV; normalized VAT (nVAT) with WMLL; nVAT with TPV; VAT/TAT ratio with WMLL, TPV, soft plaque volume, hard plaque volume and CAWT; WMLL with TPV and with soft plaque and hard plaque volume; CAWT with TPV and with soft plaque and hard plaque volume. The $\mathrm{p}$ value required for significance was calculated by applying the Bonferroni correction for multiple comparisons to achieve a global significance level of $5 \%$. Due to the small sample size, a correlation analysis separated for gender could not be performed.

\section{Results}

A total of 25 patients were studied ( 8 female, 17 male). The average age was 64.1 years \pm 11.6 . The detailed numerical scores of the following analyses are depicted in table 1 .

\section{Plaque Volume of the Aorta and the Supra-Aortic} Vessels

The mean TPV was $1,211 \mathrm{~mm}^{3}$, ranging from $0 \mathrm{~mm}^{3}$ to $6,017 \mathrm{~mm}^{3}$.

\section{Abdominal Adipose Tissue Volume}

The average TAT volume was 14.7 liters \pm 5.8 , the mean volume of SAT was $8.6 \pm 5.0$ liters and the mean volume of VAT was $6.2 \pm 2.6$ liters. The standard deviation (SD) over SAT and VAT for all subjects represents the intrasubject variability (not the mean analysis error) and is in the order of magnitude with a larger subject population in an inhouse study with $>200$ subjects (data acquisition and analysis with the identical MRI protocol, unpublished data). The estimated analysis error in determination of SAT and VAT is in the order of $10 \%$. The mean value of VAT as a percentage of TAT (VAT/TAT) was $43.5 \pm 13.5 \%$. The mean nTAT was $45.7 \pm 15.5 \mathrm{l} / \mathrm{m}$, that of nSAT was $26.3 \pm$ $13.6 \mathrm{l} / \mathrm{m}$, and the mean normalized volume of $\mathrm{nVAT}$ was $19.5 \pm 8.3 \mathrm{l} / \mathrm{m}$.

\section{White Matter Lesion Load}

The average WMLL was $6,379 \pm 7,037 \mathrm{~mm}^{3}$, ranging from 91 to $28,060 \mathrm{~mm}^{3}$.

\section{Carotid Artery Wall Thickness \\ Mean CAWT was $1.20 \pm 0.14 \mathrm{~mm}$.}

\section{Correlation Analysis}

The nTAT and the nSAT showed no significant correlation either with WMLL or TPV (fig. 2a, b). In contrast, the nVAT showed a significant correlation with WMLL volume ( $\mathrm{p}=0.001$, fig. $2 \mathrm{f}$ ) but not with TPV. VAT/TAT ratio (VAT as a percentage of TAT) showed a significant correlation with WMLL ( $\mathrm{p}=0.002$, fig. $2 \mathrm{~d})$, TPV ( $<<0.001$, fig. $2 \mathrm{c}$ ) and soft plaque volume $(\mathrm{p}<0.001)$ but not with hard plaque volume. In addition, VAT/TAT ratio showed a significant correlation with CAWT ( $p<0.001$, fig. $2 \mathrm{e}$ ).

TPV and WMLL were significantly positively correlated ( $\mathrm{p}<0.001$, table 2$)$. Furthermore, the volume of both soft and hard (calcified) plaques correlated significantly with WMLL ( $\mathrm{p}=0.003$ and $\mathrm{p}=0.001$, respectively).

CAWT was significantly positively correlated with TPV $(\mathrm{p}<0.001)$ and soft plaque volume $(\mathrm{p}<0.001)$ but not with hard plaque volume.

\section{Discussion}

The aim of our study was to investigate possible correlations between the distribution of body fat and atherosclerotic changes of the brain-feeding arteries in terms of macroangiopathy in CTA imaging on the one hand and body fat distribution and leukoaraiosis as a measure of microangiopathic changes on the other hand. Our results indicate that the proportion of visceral in relation to subcutaneous fat volume determines cerebrovascular risk rather than total body fat volume.

It is assumed that leukoaraiosis represents regions of ischemic demyelination and gliosis in consequence of chronic vascular insufficiency and clinically silent in- 


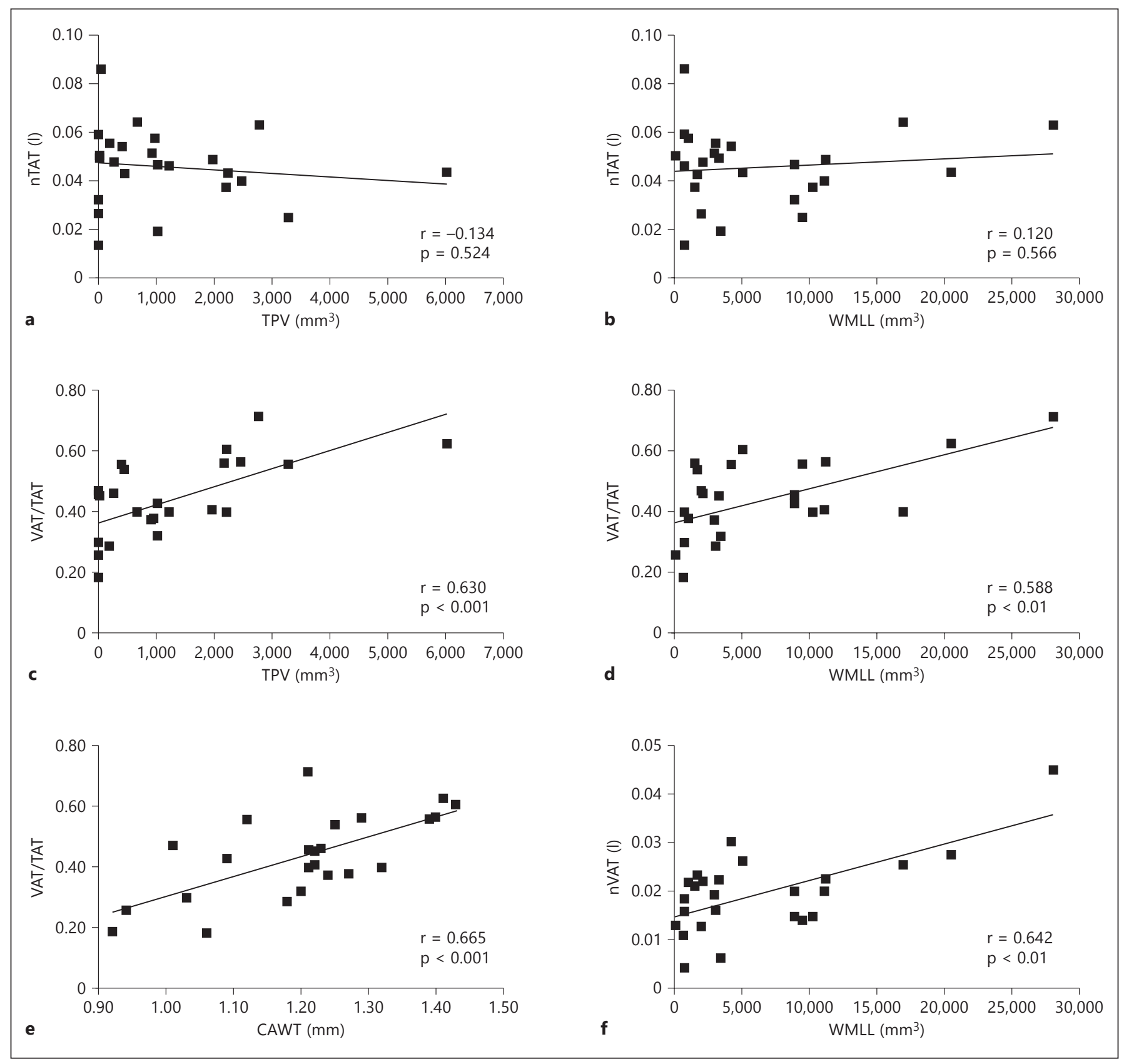

Fig. 2. a Scatterplot between nTAT volume and TPV showing no significant correlation. b Scatterplot between nTAT and WMLL, showing no significant correlation. c Positive correlation between quotient of VAT and TAT (VAT/TAT ratio) and TPV. d Positive

correlation between VAT/TAT ratio and WMLL. e Positive correlation between VAT/TAT ratio and CAWT. $\mathbf{f}$ Positive correlation between nVAT volume and WMLL.

farcts $[15,16]$. The reason for this chronic ischemia seems to be arteriolar disease which is again caused by lipohyalinosis of the media and thickening of the arteriolar wall, resulting in narrowing of the lumen of small penetrating vessels in the white matter [17]. Besides age

$[15,18]$, cerebrovascular risk factors are associated with leukoaraiosis [19]. Hypertension is the most important and most common risk factor for WMLL [20] and it has been shown that WMLL progression can be stopped or delayed by an active blood pressure-lowering regimen 
Table 2. Correlation analyses

\begin{tabular}{lccc}
\hline Test & $\begin{array}{l}\text { Pearson's } \\
\text { correlation } \\
\text { coefficient }\end{array}$ & p value & $\mathrm{n}$ \\
& 0.120 & 0.566 & 25 \\
\hline nTAT and WMLL & -0.134 & 0.524 & 25 \\
nTAT and TPV & -0.256 & 0.218 & 25 \\
\hline nSAT and WMLL & -0.401 & 0.047 & 25 \\
nSAT and TPV & 0.642 & $0.001^{\mathrm{a}}$ & 25 \\
nVAT and WMLL & 0.406 & 0.044 & 25 \\
nVAT and TPV & 0.588 & $0.002^{\mathrm{a}}$ & 25 \\
\hline VAT/TAT and WMLL & 0.630 & $<0.001^{\mathrm{a}}$ & 25 \\
VAT/TAT and TPV & 0.672 & $<0.001^{\mathrm{a}}$ & 25 \\
VAT/TAT and soft plaque volume & 0.463 & 0.020 & 25 \\
VAT/TAT and hard plaque volume & 0.665 & $<0.001^{\mathrm{a}}$ & 25 \\
VAT/TAT and CAWT & 0.665 & $<0.001^{\mathrm{a}}$ & 25 \\
\hline WMLL and TPV & 0.660 & $0.003^{\mathrm{a}}$ & 25 \\
WMLL and soft plaque volume & 0.570 & $0.001^{\mathrm{a}}$ & 25 \\
WMLL and hard plaque volume & 0.627 & $<0.001^{\mathrm{a}}$ & 25 \\
\hline CAWT and TPV & 0.674 & 0.014 & 25 \\
CAWT and soft plaque volume & 0.725 & 0.487 & \\
CAWT and hard plaque volume & $0.01^{\mathrm{a}}$ & 25 \\
\hline
\end{tabular}

a Significant after Bonferroni correction.

[21]. However, other common risk factors for cerebrovascular disease like diabetes have also been proposed $[17,22,23]$. In a study by Rost et al. [24], only age and elevated homocysteine levels were independently associated with WMLL volume in patients with acute ischemic stroke, suggesting that WMLL burden in patients with stroke may not only be mediated by traditional vascular risk factors and that there may be a difference in the development of WMLL between the general population and patients with stroke. The associations between distribution patterns of adiposity and leukoaraiosis have not been studied previously according to our knowledge. Our results indicate that a higher percentage of visceral fat may be an important risk factor for the development of leukoaraiosis. Further studies have to show if this association is mediated by adiposity-related risk factors for leukoaraiosis such as hypertension or diabetes mellitus, or if a higher percentage of VAT is an independent risk factor by itself.

With regard to correlations of atherosclerosis and leukoaraiosis, previous studies have demonstrated significant correlations between the degree of carotid stenosis and the degree of leukoaraiosis, but showed controversial results about the correlation between plaque load and severity of white matter disease [25]. Breteler et al. [26] showed that WMLL is associated with atherosclerosis (i.e. increased carotid intima media thickness and carotid plaques). De Leeuw et al. [27] were able to demonstrate that atherosclerosis of the aorta did significantly correlate with periventricular white matter hyperintensities. In other studies, it has been shown that an increased cardiac, peripheral arterial and carotid arterial atherosclerosis is associated with leukoaraiosis $[20,28]$. Other studies could not demonstrate a significant correlation between calcified plaques and leukoaraiosis, but only a statistical trend toward increased leukoaraiosis in patients with predominantly fatty carotid plaques [25].

Our results indicate that a high percentage of visceral fat may have an effect on WMLL and on atherosclerotic plaque load, so that a higher percentage of visceral fat might be seen as a risk factor for both micro- and macroangiopathic vascular changes. We also found significant correlations for total plaque load and soft and hard plaque volume with WMLL. It is not clear if the effects of visceral adiposity on atherosclerosis leads to increased WMLL in subjects with increased visceral fat volume or if visceral adiposity has an independent effect on WMLL and on plaque load.

The effect of body fat distribution on leukoaraiosis may be caused by its effect on atherosclerosis to some extent, especially by subtle changes in atherosclerotic plaque configuration. For example, Altaf et al. [29] could show a positive correlation between unstable carotid plaques and the number of leukoaraiotic lesions as well as between white matter hyperintensities and intraplaque hemorrhage. Presumably an even more precise differentiation of the composition of plaques would be helpful in further studies, especially as the activity of atherosclerotic plaques may be important for the development of leukoaraiosis.

Associations between adiposity and coronary or cerebrovascular atherosclerosis have been demonstrated in several studies just recently. BMI in young men has been shown to be associated with coronary atherosclerosis in terms of fatty streaks, raised lesions and stenosis in the coronary arteries [30]. Likewise, an increase in visceral fat is significantly associated with noncalcified coronary plaque burden [31] and progression of noncalcified coronary plaques [32]. Yu et al. [33] found that abdominal obesity by itself is an independent marker of subclinical atherosclerosis in women. In other studies, abdominal obesity was also associated with arterial stiffness [34] and an increase in carotid intima media thickness $[34,35]$. 
Konishi et al. [36] showed that the visceral fat area is associated with carotid artery plaque formation in type 2 diabetes.

Our study assesses both the volume and distribution of body fat and plaque volumes with the result of a close correlation between these variables, and our data reveal that a higher percentage of VAT has an effect on atherosclerosis of the brain-feeding arteries, as indicated by an increase in CAWT and a higher TPV. In particular, the soft (fibrous or fatty) component of plaques is increased by visceral adiposity, as VAT/TAT ratio has a stronger association with the volume of soft rather than hard plaques.

Much effort has been made in recent years to better understand the link between abdominal adiposity and the metabolic syndrome and how visceral adiposity influences cardiovascular risk. It has been found that VAT leads to an altered metabolic profile with an insulin resistant, proinflammatory, prothrombotic and prohypertensive state [4], probably by the release of adipokines and inflammatory cytokines like interleukin-6 and TNFa [5] and by an altered free fatty acid metabolism in the portal circulation [37]. Our data give strong support to the assumption that the fatal consequences of visceral adiposity [30-32] are not limited to CVD but also directly influence cerebral circulation and thereby cerebrovascular disease.

\section{Conclusion}

Our results contribute to existing studies about the association of different patterns of fat distribution with atherosclerosis in general and with atherosclerosis of the brain-feeding arteries in particular [35, 36], highlighting the importance of visceral adiposity as a risk factor for cerebrovascular disease. Furthermore, visceral adiposity not only contributes to macroangiopathic changes of the vessels, but also seems to induce microangiopathic changes in terms of a higher load of white matter lesions. The percentage of VAT in TAT (VAT/TAT ratio) has the potential of a sensitive parameter and might become a relevant new epidemiological marker, showing highly significant correlations with well-established markers of cerebrovascular disease. In conclusion, the percentage of VAT by itself has to be regarded as a risk factor for both small vessel cerebrovascular disease and cerebral atherosclerosis of the large-to-medium-sized arteries. Thus, fat distribution patterns with a high percentage of VAT should be considered a high-risk form of obesity, contributing to vascular dementia and ischemic stroke.

\section{Disclosure Statement}

All authors declare that they have no conflicts of interest.

\section{References}

1 Behrouz R, Malek AR, Torbey MT: Small vessel cerebrovascular disease: the past, present, and future. Stroke Res Treat 2012;2012: 839151.

2 Mono ML, Karameshev A, Slotboom J, Remonda L, Galimanis A, Jung S, Findling O, de Marchis GM, Luedi R, Kiefer C, Stuker C, Mattle HP, Schroth G, Arnold M, Nedeltchev K, El-Koussy M: Plaque characteristics of asymptomatic carotid stenosis and risk of stroke. Cerebrovasc Dis 2012;34: 343-350.

-3 Zhang CE, van Raak EP, Rouhl RP, Lodder J, Staals J, Knottnerus IL, van Oostenbrugge RJ: Metabolic syndrome relates to lacunar stroke without white matter lesions: a study in firstever lacunar stroke patients. Cerebrovasc Dis 2010;29:503-507.

-4 Despres JP, Arsenault BJ, Cote M, Cartier A, Lemieux I: Abdominal obesity: the cholesterol of the 21st century? Can J Cardiol 2008; 24(suppl D):7D-12D.
5 Hotamisligil GS: Molecular mechanisms of insulin resistance and the role of the adipocyte. Int J Obes Relat Metab Disord 2000; 24(suppl 4):S23-S27.

$\checkmark 6$ Rondinone CM: Adipocyte-derived hormones, cytokines, and mediators. Endocrine 2006;29:81-90.

7 Kissebah AH: Intra-abdominal fat: is it a major factor in developing diabetes and coronary artery disease? Diabetes Res Clin Pract 1996; 30(suppl):25-30.

-8 Kurth T, Gaziano JM, Berger K, Kase CS, Rexrode KM, Cook NR, Buring JE, Manson JE: Body mass index and the risk of stroke in men. Arch Intern Med 2002;162:2557-2562.

-9 Lu M, Ye W, Adami HO, Weiderpass E: Prospective study of body size and risk for stroke amongst women below age 60. J Intern Med 2006;260:442-450.

10 Winter Y, Rohrmann S, Linseisen J, Lanczik $\mathrm{O}$, Ringleb PA, Hebebrand J, Back T: Contribution of obesity and abdominal fat mass to risk of stroke and transient ischemic attacks. Stroke 2008;39:3145-3151.
1 Hu G, Tuomilehto J, Silventoinen K, Sarti C, Mannisto S, Jousilahti P: Body mass index, waist circumference, and waist-hip ratio on the risk of total and type-specific stroke. Arch Intern Med 2007;167:1420-1427.

12 Saba L, Sanfilippo R, Montisci R, Mallarini G: Associations between carotid artery wall thickness and cardiovascular risk factors using multidetector CT. AJNR Am J Neuroradiol 2010;31:1758-1763.

13 Muller HP, Raudies F, Unrath A, Neumann $\mathrm{H}$, Ludolph AC, Kassubek J: Quantification of human body fat tissue percentage by MRI. NMR Biomed 2011;24:17-24.

14 Muller HP, Unrath A, Ludolph AC, Kassubek J: Preservation of diffusion tensor properties during spatial normalization by use of tensor imaging and fibre tracking on a normal brain database. Phys Med Biol 2007;52:N99-N109.

15 Ovbiagele B, Saver JL: Cerebral white matter hyperintensities on MRI: current concepts and therapeutic implications. Cerebrovasc Dis 2006;22:83-90. 
16 LADIS Study Group: 2001-2011: a decade of the LADIS (Leukoaraiosis And Disability) Study: what have we learned about white matter changes and small-vessel disease? Cerebrovasc Dis 2011;32:577-588.

17 Pantoni L, Garcia JH: The significance of cerebral white matter abnormalities 100 years after Binswanger's report. A review. Stroke 1995;26:1293-1301.

18 Ylikoski A, Erkinjuntti T, Raininko R, Sarna S, Sulkava R, Tilvis R: White matter hyperintensities on MRI in the neurologically nondiseased elderly. Analysis of cohorts of consecutive subjects aged 55 to 85 years living at home. Stroke 1995;26:1171-1177.

19 Jeerakathil T, Wolf PA, Beiser A, Massaro J, Seshadri S, D’Agostino RB, DeCarli C: Stroke risk profile predicts white matter hyperintensity volume: the Framingham Study. Stroke 2004;35:1857-1861.

20 Melkas S, Sibolt G, Oksala NK, Putaala J, Pohjasvaara T, Kaste M, Karhunen PJ, Erkinjuntti T: Extensive white matter changes predict stroke recurrence up to 5 years after a first-ever ischemic stroke. Cerebrovasc Dis 2012;34:191-198.

21 Dufouil C, Chalmers J, Coskun O, Besancon V, Bousser MG, Guillon P, MacMahon S, Mazoyer B, Neal B, Woodward M, Tzourio-Mazoyer $\mathrm{N}$, Tzourio C: Effects of blood pressure lowering on cerebral white matter hyperintensities in patients with stroke: the PROGRESS (Perindopril Protection Against Recurrent Stroke Study) Magnetic Resonance Imaging Substudy. Circulation 2005;112:1644-1650.

-22 Dufouil C, de Kersaint-Gilly A, Besancon V, Levy C, Auffray E, Brunnereau L, Alperovitch A, Tzourio C: Longitudinal study of blood pressure and white matter hyperintensities: the EVA MRI cohort. Neurology 2001;56: 921-926.
23 Fazekas F, Niederkorn K, Schmidt R, Offenbacher H, Horner S, Bertha G, Lechner H: White matter signal abnormalities in normal individuals: correlation with carotid ultrasonography, cerebral blood flow measurements, and cerebrovascular risk factors. Stroke 1988; 19:1285-1288.

24 Rost NS, Rahman R, Sonni S, Kanakis A, Butler C, Massasa E, Cloonan L, Gilson A, Delgado P, Chang Y, Biffi A, Jimenez-Conde J, Besanger A, Silva G, Smith EE, Rosand J, Furie KL: Determinants of white matter hyperintensity volume in patients with acute ischemic stroke. J Stroke Cerebrovasc Dis 2010;19:230235.

25 Saba L, Sanfilippo R, Pascalis L, Montisci R, Mallarini G: Carotid artery abnormalities and leukoaraiosis in elderly patients: evaluation with MDCT. AJR Am J Roentgenol 2009; 192:W63-W70.

26 Breteler MM, van Swieten JC, Bots ML, Grobbee DE, Claus JJ, van den Hout JH, van Harskamp F, Tanghe HL, de Jong PT, van Gijn J, et al: Cerebral white matter lesions, vascular risk factors, and cognitive function in a population-based study: the Rotterdam Study. Neurology 1994;44:1246-1252.

27 De Leeuw FE, de Groot JC, Oudkerk M, Witteman JC, Hofman A, van Gijn J, Breteler MM: Aortic atherosclerosis at middle age predicts cerebral white matter lesions in the elderly. Stroke 2000;31:425-429.

28 Bots ML, van Swieten JC, Breteler MM, de Jong PT, van Gijn J, Hofman A, Grobbee DE: Cerebral white matter lesions and atherosclerosis in the Rotterdam Study. Lancet 1993; 341:1232-1237.

29 Altaf N, Morgan PS, Moody A, MacSweeney ST, Gladman JR, Auer DP: Brain white matter hyperintensities are associated with carotid intraplaque hemorrhage. Radiology 2008; 248:202-209.

-30 McGill HC Jr, McMahan CA, Herderick EE, Zieske AW, Malcom GT, Tracy RE, Strong JP: Obesity accelerates the progression of coronary atherosclerosis in young men. Circulation 2002; 105:2712-2718.
31 Ohashi N, Yamamoto H, Horiguchi J, Kitagawa T, Kunita E, Utsunomiya H, Oka T, Kohno N, Kihara Y: Association between visceral adipose tissue area and coronary plaque morphology assessed by CT angiography. JACC Cardiovasc Imaging 2010;3:908-917.

32 Imai A, Komatsu S, Ohara T, Kamata T, Yoshida J, Miyaji K, Takewa M, Kodama K: Visceral abdominal fat accumulation predicts the progression of noncalcified coronary plaque. Atherosclerosis 2012;222:524-529.

$33 \mathrm{Yu} \mathrm{RH}, \mathrm{Ho}$ SC, Ho SS, Woo JL, Ahuja AT: Association of general and abdominal obesities and metabolic syndrome with subclinical atherosclerosis in asymptomatic Chinese postmenopausal women. Menopause 2008; 15: 185-192.

34 Recio-Rodriguez JI, Gomez-Marcos MA, Patino-Alonso MC, Agudo-Conde C, RodriguezSanchez E, Garcia-Ortiz L: Abdominal obesity vs general obesity for identifying arterial stiffness, subclinical atherosclerosis and wave reflection in healthy, diabetics and hypertensive. BMC Cardiovasc Disord 2012;12:3.

-35 De Michele M, Panico S, Iannuzzi A, Celentano E, Ciardullo AV, Galasso R, Sacchetti L, Zarrilli F, Bond MG, Rubba P: Association of obesity and central fat distribution with carotid artery wall thickening in middle-aged women. Stroke 2002;33:2923-2928.

36 Konishi K, Nakano S, Seto H, Tsuda S, Koya $\mathrm{D}$ : Carotid atherosclerosis mediated by visceral adiposity and adipocytokines in type 2 diabetic subjects. Diabetes Res Clin Pract 2009;85:171-178.

37 Despres JP, Lemieux I, Bergeron J, Pibarot P, Mathieu P, Larose E, Rodes-Cabau J, Bertrand OF, Poirier P: Abdominal obesity and the metabolic syndrome: contribution to global cardiometabolic risk. Arterioscler Thromb Vasc Biol 2008;28:1039-1049. 